\title{
BILATERAL APICAL FUNGAL BALL OF POST TUBERCULAR FIBROCAVITY OF LUNG
}

\author{
D. Sudeena ${ }^{1}$, S. B. Lal², B. Phani Kumar ${ }^{3}$, Sree Karuna Murthy Kolli ${ }^{4}$
}

${ }^{1}$ Associate Professor, Department of Pulmonary Medicine, Osmania Medical College, Hyderabad, Andhra Pradesh.

${ }^{2}$ Registrar, Dr. NTR University of Health Sciences, Vijayawada \& Former Professor \& HOD of Pulmonary Medicine, Siddhartha Medical College, Vijayawada, Andhra Pradesh.

${ }^{3}$ Assistant Professor, Department of Pulmonary Medicine, Govt. General \& Chest Hospital, Irramnuma, Hyderabad. ${ }^{4}$ Associate Professor, Department of Community, Dr. PSIMS and RF, Krishna District.

\begin{abstract}
A 60 year female, housewife, presented with recurrent cough, breathlessness, hemoptysis from the past 20 yrs. Hemoptysis turned severe since 6 yrs., along with expectaration. Her family history is not significant. Patient has typical clubbing of all digits. On examination of chest, there is B/L wasting of muscles and increased hollowness of apical chest, trachea in midline, apical impulse normal in $5^{\text {th }}$ left ICS. Vocal tactile fremitus decreased on B/L supra and infraclavicular areas, impaired note present. Fine mid inspiratory crepts, not altered with cough. No post tussive suction noted. Bronchial breath sounds present with decreased VR, due to a fungal ball occupying B/L apical fibrocavities. Patient maintaining normal oxygen saturation, but during bouts of Hemoptysis, there is a fall in oxygen saturation, along with breathlessness. Sputum for afb negative at RNTCP (1) culture of fungal elements positive. FOB BAL fluid sent for culture. Aspergillus fumigates is isolated. On Trendelenberg position, CXR revealed B/L apical fibrocavity with fungal ball. HRCT revealed B/L apical fibrocavity with fungal ball with air crescent sign positive on both sides.
\end{abstract}

KEYWORDS: Bilateral Apical Fungal Ball.

HOW TO CITE THIS ARTICLE:D. Sudeena, S. B. Lal, B. Phani Kumar, Sree Karuna Murthy Kolli. "Bilateral Apical Fungal Ball of Post Tubercular Fibrocavity of Lung". Journal of Evolution of Medical and Dental Sciences 2015; Vol. 4, Issue 92, November 16; Page: 15728-15730, DOI: $10.14260 /$ jemds/2015/2269.

INTRODUCTION: Fungal ball definition: Also known as Aspergilloma.(1)(2)(3) or Mycetoma, is a clump of mould which exist in a body cavity, such as paranasal sinuses or an organ like lung.

Prevalence: $25-80 \%$ depending on prevalence of TB.(4)

CASE REPORT: A 60 yr. old female, a housewife, presented with severe episodes of hemoptysis since 5-6 yrs. 3-4 episodes in a year, with bouts of severe hemoptysis. She suffered with T. B.(5) took short course daily regimen chemotherapy, not under observation by Govt. of TB drugs. She used Rifampicin, INH, Ethambutol, Pyrazinamide and Inj. Rifampicin on and off, irregularly for 4 months. She did not complete the course. She is a known hypertensive, Diabetic. Her cardiac status normal. On Respiratory system examination, B/L apical bronchial breath sounds with fine mid inspiratory crepts heard on both supra and infra clavicular areas anteriorly. Per abdomen Liver is normal. On CVS examination. S1, S2 normal, no murmurs.

- CXR PA view showed B/L apical fibrocavity with fungal ball.(5)bilaterally, with remaining lung parenchyma normally aerated.

- HRCT confirmed B/L fibrocavity with air crescent fungal balls.(6) bilaterally.

Financial or Other, Competing Interest: None. Submission 03-10-2015, Peer Review 04-10-2015,

Acceptance 20-10-2015, Published 13-11-2015.

Corresponding Author:

Dr. D. Sudeena,

Associate Professor, Department of Pulmonary Medicine,

Osmania Medical College, Hyderabad, India.

E-mail: drsudeenaravi@gmail.com

DOI:10.14260/jemds/2015/2269.
- FOB done, BAL collected, sent for fungal culture. Isolated Aspergillus fumigates.(6)

- Sputum AFB - negative.

- Fungal culture of sputum- negative.

- Gram stain of sputum- negative.

- ECG- NAD.

TREATMENT: The patient treated with antifungal agents itraconazole $250 \mathrm{mg}$ tid for 1 weekfollowed by od for 2 weeks and the patient was on regular follow up and intermittent therapy with itraconazole was given for 3 months. hemoptysis episodes with suitable antibiotics, bed rest, cough suppressants, antistyptics like ethamsylate and tranexenic acid and physiotherapy. Blood transfusion done, as per requirement. freqency of hemoptysis decreased. symptoms relieved and She was explained of her condition and the prognosis.

DISCUSSION: Post tubercular patient with irregular Antituberculosis treatment complicated with Fungal ball.(7) bilaterally. The bouts of hemoptysis being severe, the patient is hospitalized repeatedly, 3-4 times per year. The severity of symptoms, as per her age, the condition of her, is poor, and prognosis is explained to her relatives.

Though India is the second populous country in the World, $1 / 4^{\text {th }}$ of the global incident TB.(2) cases occur in India annually. TB is a serious public health problem in India, as it runs first among 22 high burden countries causing immense morbidity and mortality. Several factors have been identified, including the individuals perception of disease, socio economic level, extent of awareness about the disease and DOTS centre.

This was carried to assess awareness about TB disease, spread, treatment, prevention. 
TB.(3) is more prevalent in men than in women, maybe, due to biological vulnerability of men to infections or exposure at work place. $71 \%$ were from the economically productive age group.

Aspergillus.(5) fumigatus $58 \%-64 \%$ of post tubercular is the commonest occurrence of fungal elements isolated. It is also common known for secondary colonization in lung Cavities, bronchiectatic cavities, lung absesses, lung cysts, malignant persistent cavity. Fungal ball is a discrete lesion, that classically colonizers of healed pulmonary TB.(6) and other fibrotic lung diseases.

Only $51 \%$ of the doctors were aware that $\mathrm{TB}$ is commonly complicated with fungal disorders. Delays in diagnosis because of unusually clinical or radiographic presentated constitute a significant public health hazard.

CONCLUSION: All the awareness regarding basic aspects of TB was adequate. Knowledge about prevention, curability, and free treatment of TB.(7) should be stressed. Periodis KAP (Knowledge, attitude and practice) surveys will help in identifying the deficiencies in knowledge and practices of the community, thereby providing clues to improve the performance of RNTCP.

ACKNOWLEDGEMENTS: The Authors, hereby, thank the Postgraduate students of names, Dr. G. Vedaranya, of Gandhi Medical College, and Dr. T. Keerthi, of Osmania Mdical College, and Microbiology dept. and RNTCP.(1) staff, Radiology Dept. of Osmania Medical College, for their assistance during the study.

\section{REFERENCES:}

1. Leon EE, Craig TJ: Antifungals in the treatment of Allergic bronchopulmonary aspergillosis. Ann Allergy Asthma Immunol 1999, 82:511-517

2. Izidor Kern and Anton Lopert: Allergic bronchopulmonary aspergillosis with coexistant aspergilloma: a case report. Journal of Medical Case reports. 2010,4:309

3. Mansi Gupta et al. Allergic bronchopulmonary aspergillosis without asthma complicating PTB. Lung India 2012, 3; 286288

4. Rajeswari R, Balasubramanian M, Muniyandi S, Geetharamani S, Theresan X. Socioeconomic impact of TB on patients and family in India. Int. J. tubercul lung dis. 3(10):869-877

5. Wandwalo E.R. \& O. Merkve. Knowledge of disease and treatment among TB patients in Mwanza, Tanania International journal of TB \& lung disease. 2000; 4(11): 1041-46

6. Malhotra, Taneja DK, Dhingra VK, Rajpal S, Mehra M. Awareness regarding TB in a rural population of Delhi. Ind J Comm Med. 2002; 27:62-8

7. TB INDIA 2014 Revised National TB Control Programme ANNUAL STATUS REPORT.

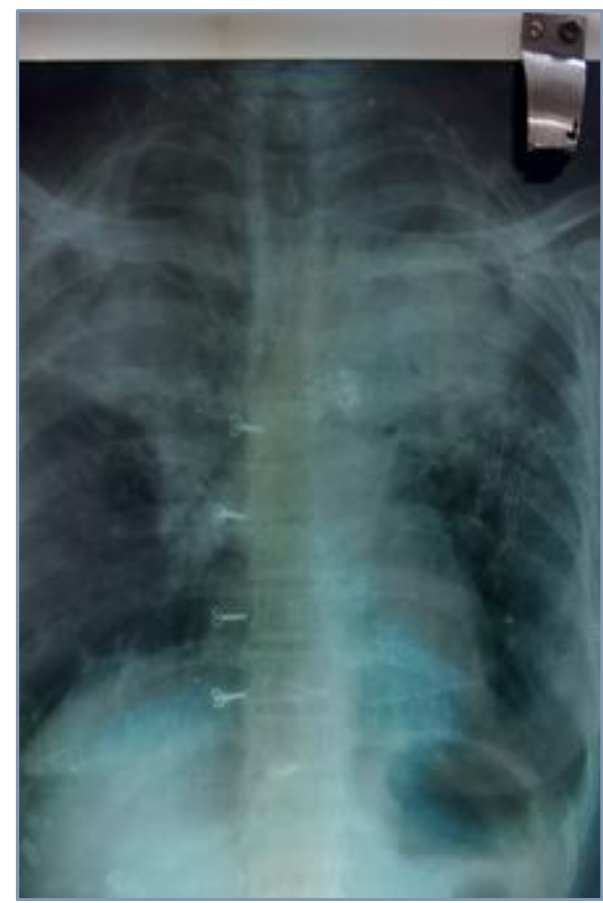

Bilateral Apical Fungal Balls 


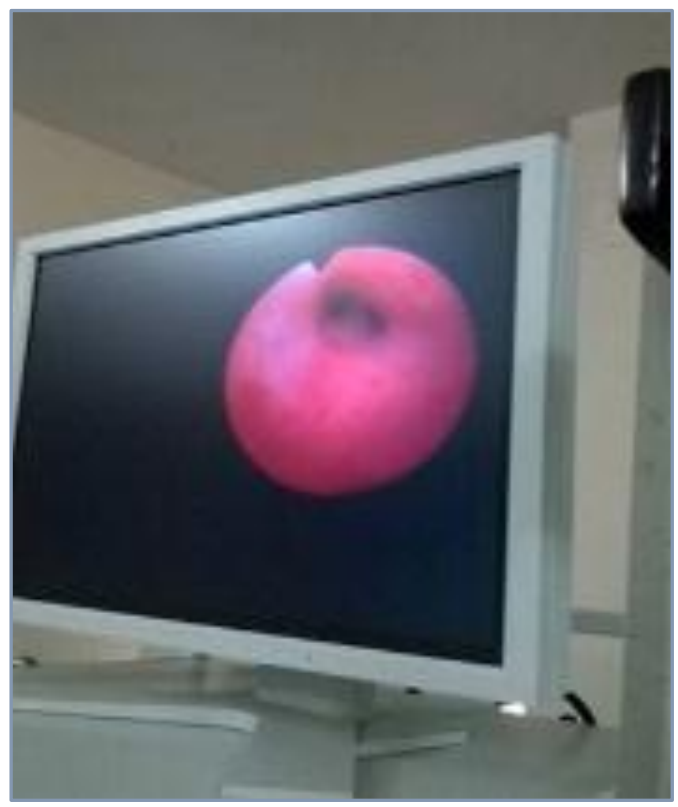

Erethymatous and Edematous Mucosa seen over right and left main bronchus 